\title{
Analysis of coptisine, berberine and palmatine in adulterated Chinese medicine by capillary electrophoresis-electrospray ion trap mass spectrometry
}

\author{
Yet Ran Chen ${ }^{\mathrm{a}}$, Kuo Ching Wen ${ }^{\mathrm{b}}$, Guor Rong Her ${ }^{\mathrm{a}, *}$ \\ ${ }^{a}$ Department of Chemistry, National Taiwan University, Taipei, Taiwan \\ ${ }^{\mathrm{b}}$ National Laboratories of Food and Drugs, Department of Health, Executive Yuan, Taipei, Taiwan
}

Received 1 June 1999; received in revised form 11 October 1999; accepted 13 October 1999

\begin{abstract}
Chinese medicine preparations contaminated with coptisine, berberine and palmatine were studied by capillary electrophoresis-electrospray ion trap mass spectrometry. The dubious adulterants were identified by their retention times, molecular ions and specific fragment ions produced from collision induced dissociation. The results showed that, in comparison with CE-UV and capillary electrophoresis-electrospray mass spectrometry (CE-ESI-MS), more reliable identification could be achieved with CE-ESI-MS-MS using ion trap mass spectrometry. (c) 2000 Elsevier Science B.V. All rights reserved.
\end{abstract}

Keywords: Pharmaceutical analysis; Coptisine; Berberine; Palmatine; Alkaloids

\section{Introduction}

It is not very uncommon to see traditional Chinese medicine illegally adulterated by the addition of synthetic chemical drugs. Recently, methods based on high-performance liquid chromatography (HPLC) [1-12] and capillary electrophoresis (CE) [13-20] have been developed for the analysis of these adulterants in Chinese medicine. In comparison with HPLC, CE often provides excellent separation efficiency and short analysis time. However, owing to the often-unavoidable capillary surface modification resulting from the complex Chinese medicine preparation, reproducible migration times are difficult to

\footnotetext{
*Corresponding author. Tel.: +886-2-2369-0152 ext. 109; fax: $+886-2-2363-8058$.
}

obtain and thus reliable compound identification is difficult.

Because of its low detection limit, high specificity and more importantly abundant structural information, mass spectrometry (MS) has been considered as one of the ideal methods for chromatographic detection [21-23]. The merits of using MS as the chromatographic detector are best demonstrated with the highly successful gas chromatography (GC)-MS. The coupling of HPLC or CE with MS has been a much more difficult task than the interfacing of GC with MS; however, recent developments in MS such as electrospray ionization (ESI) [24-27] and atmospheric pressure chemical ionization (APCI) make the coupling of CE with MS much easier [28-32].

In Taiwan, the herbs used in the preparation of Chinese medicine have to be approved and also 
labeled clearly on the bottle. It is illegal to include any Chinese herb which is not listed on the package. A Chinese medicine, wuyoufun-13, was suspected to contain coptisine, berberine and palmatine. These compounds are active ingredients of Coptidis rhizoma with antibacterial [33] and anticonvulsant [34] activity. This preparation was studied by CEUV, CE-ESI-MS and CE-ESI-MS-MS. The merits and limitations of the different approaches are discussed.

\section{Experimental}

\subsection{Reagents and materials}

Methanol, ammonium acetate, acetic acid were of chromatographic grade from J.T. Baker (Phillipsburg, NJ, USA). Deionized (18 M $\Omega$ ) water (Milli-Q water system, Millipore, Bedford, MA, USA) was used in the preparation of the samples and buffer solution. Berberine and palmatine chloride were obtained form Sigma (St. Louis, MO, USA), coptisine chloride was obtained from Nacalai Tesque (Kyoto, Japan). Coptisine, berberine and palmatine standard solutions were prepared by dissolving 1.2 $\mathrm{mg}$ powder in $5 \mathrm{ml}$ of $70 \%$ aqueous methanol.

\subsection{Preparation of sample extract}

Powder of "wuyoufun-13" was purchased from a local drug store in Taipei. A sample of ca. $10 \mathrm{~g}$ was extracted with $50 \mathrm{ml} 70 \%$ methanol and filtered with No. 1 filter paper. The extract was dried and dissolved in $5 \mathrm{ml} \mathrm{70 \%} \mathrm{methanol} \mathrm{and} \mathrm{filtered} \mathrm{with} \mathrm{a}$ $0.45-\mu \mathrm{m}$ filter.

\subsection{CE-UV system}

The CE system was constructed in the laboratory and had been described elsewhere [35]. CE columns were fused-silica capillaries (Polymicro Technologies, Phoenix, AZ, USA) of $85 \mathrm{~cm}(70 \mathrm{~cm}$ to the detector $) \times 50 \mu \mathrm{m}$ I.D. $\times 375 \mu \mathrm{m}$ O.D. A small area of the polyimide coating was burned off to form a window for UV detection. On column detection was performed on an UV detector (UV-C Rainin, Emeryville, CA, USA) operated at $230 \mathrm{~nm}$. The capillary column was washed with $1 M \mathrm{NaOH}$ followed by $0.1 M \mathrm{NaOH}$, water and running buffer. The capillary column was equilibrated with running buffer for $20 \mathrm{~min}$ before each run.

\subsection{Mass spectrometry}

A Finnigan LCQ quadrupole ion trap mass spectrometer equipped with an electrospray ionization source (Finnigan MAT, San Jose, CA, USA) was used. The spray voltage was set to $+4.5 \mathrm{kV}$ and the capillary temperature was set to $200^{\circ} \mathrm{C}$. Standards were infused with a syringe pump at a flow-rate of 5 $\mu \mathrm{l} / \mathrm{min}$.

The CE-MS interface utilizes a triaxial flow arrangement whereby CE eluent is mixed with a suitable sheath liquid at the tip and nebulized by nitrogen gas. CE columns were $70 \mathrm{~cm}$ long. High voltage applied on the buffer reservoir was $24.5 \mathrm{kV}$. With a $+4.5 \mathrm{kV}$ ESI voltage applied on the outlet of the separation column, the potential difference during the analysis was about $20 \mathrm{kV}$ for CE-ESI-MS analysis. For the purpose of electrical contact, a sheath liquid was delivered at a flow-rate of $5 \mu \mathrm{l} /$ min by a syringe pump. The mass spectrometer was operated in the positive ion mode and data were collected by selected ion monitoring (SIM) for CE-

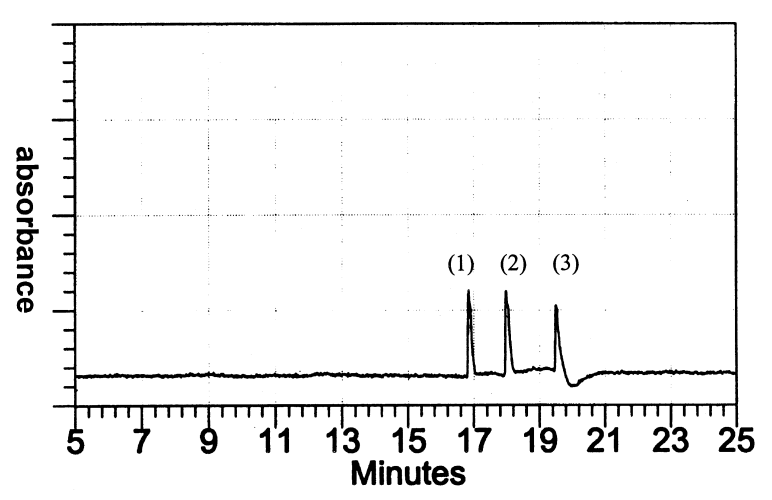

Fig. 1. CE-UV electropherogram of coptisine, berberine and palmatine. A $85 \mathrm{~cm}(70 \mathrm{~cm}$ effective length $) \times 50 \mu \mathrm{m}$ I.D. fusedsilica capillary tubing was used with $50 \mathrm{~m} M$ ammonium acetate at $\mathrm{pH} 3.8$ as running buffer. The potential was $+20 \mathrm{kV}$ and the detection wavelength was $230 \mathrm{~nm}$. Sample (33 ppm) was injected hydrodynamically 15 mbar for $10 \mathrm{~s}$. Peaks were assigned as (1) coptisine, (2) berberine and (3) palmatine. 


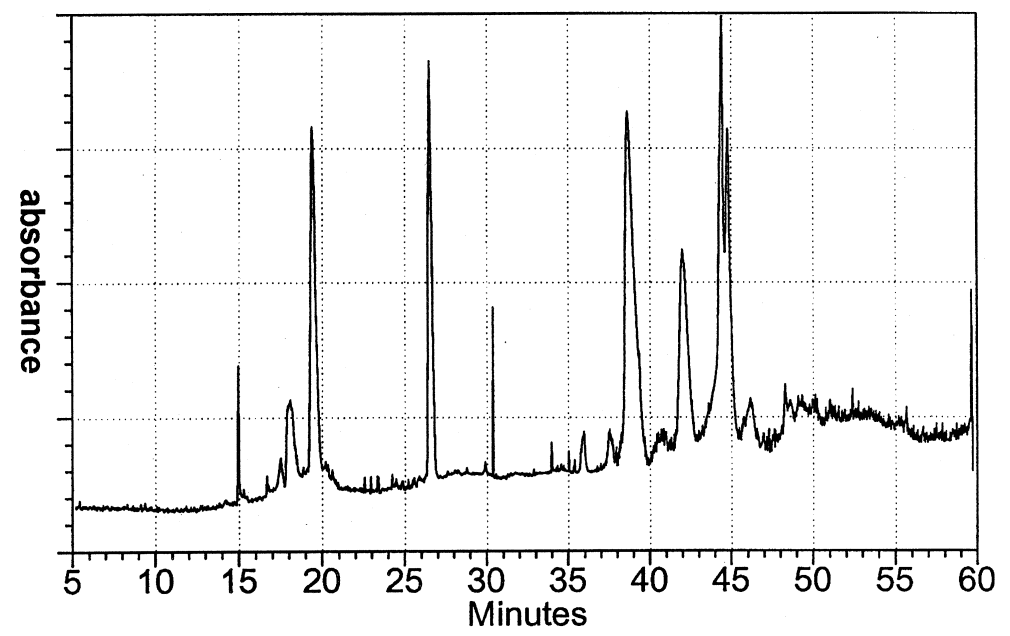

Fig. 2. CE-UV electropherogram of Chinese medicine possibly contaminated with coptisine, berberine and palmatine. Conditions as in Fig. 1.

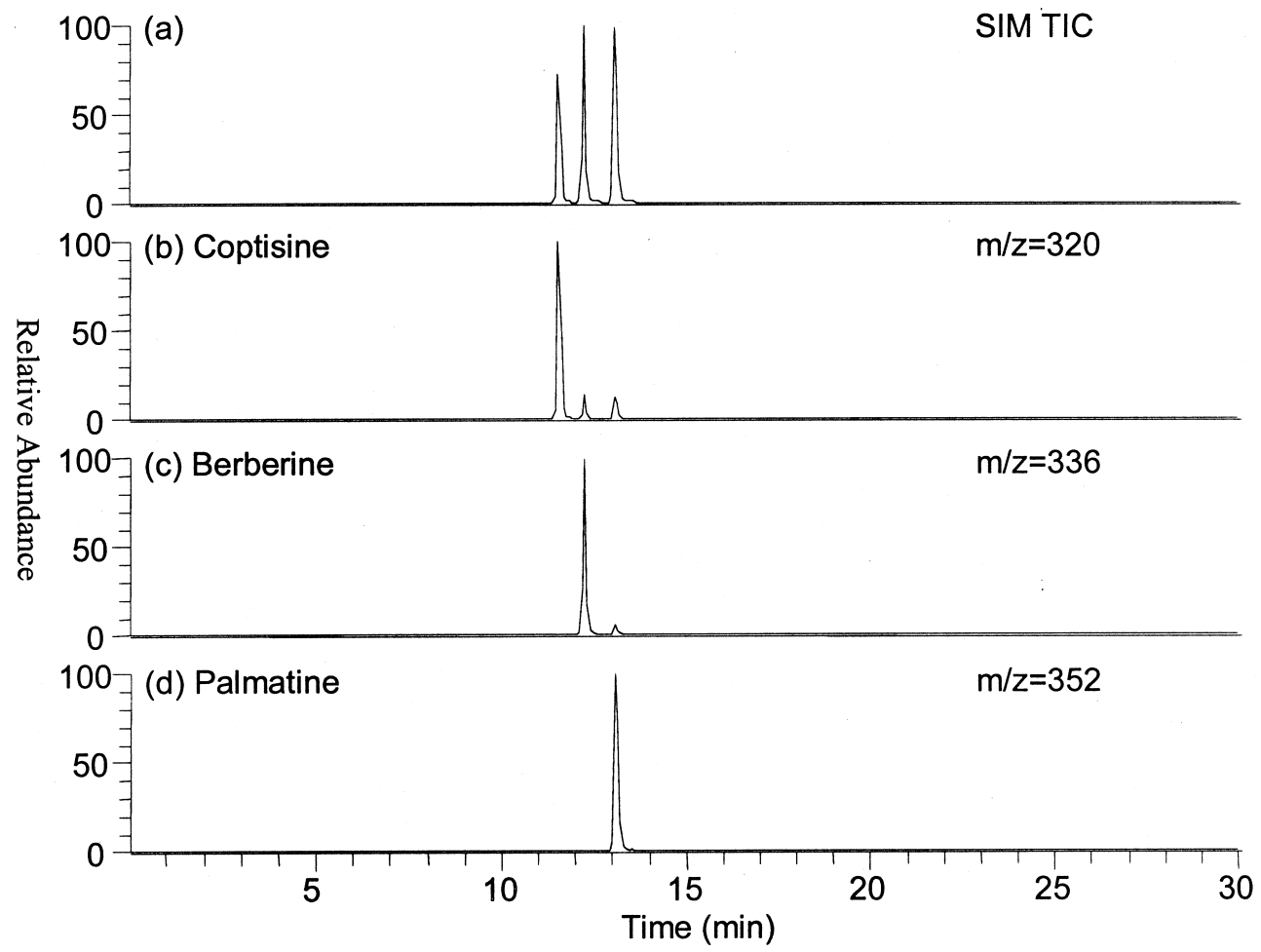

Fig. 3. CE-ESI-MS electropherograms of coptisine, berberine and palmatine. Sheath liquid composition was methanol-water (80:20) containing $1 \%$ acetic acid. The potential used for $\mathrm{CE}$ separation was $+24 \mathrm{kV}$ and the ESI voltage was $+4.5 \mathrm{kV}$. SIM $=$ Selected ion monitoring; TIC $=$ total ion current. 
ESI-MS analysis and selected reaction monitoring (SRM) for CE-ESI-MS-MS analysis.

\section{Results and discussion}

\section{1. $C E-U V$ analysis}

CE-UV analysis of coptisine, berberine and palmatine has been reported using sodium acetate as the running buffer [20]. For ESI-MS analysis, sodium acetate was replaced with a more volatile buffer, ammonium acetate. As can be seen, coptisine, berberine and palmatine were baseline resolved by $\mathrm{CE}$ using ammonium acetate as the running buffer (Fig. 1). CE-UV analysis of the crude extract that was possibly contaminated with small amount of coptisine, berberine and palmatine is shown in Fig. 2 . Although the electropherogram of the extract was more complicated than the standards, two peaks with similar a retention time to berberine (about $18 \mathrm{~min}$ ) and palmatine (about $19 \mathrm{~min}$ ) were observed. The addition of authentic standards into the extract before CE-UV analysis suggests that these two peaks are very likely berberine and palmatine. Based on UV absorbance, the abundance of palmatine (about 19 min) is significantly higher than berberine (about 18 min). Two drawbacks were found resulting from the complexity of crude extract. In addition to the complexity of electropherogram which makes the assignment difficult, the migration time also changes significantly if the column is not washed extensively after each analysis. In order to provide a more conclusive answer, the extract was further studied with CE-ESI-MS.

\subsection{CE-ESI-MS and CE-ESI-MS-MS analysis}

ESI is a soft ionization method and the ESI mass spectra of coptisine, berberine and palmatine are characterized with molecular ions with fragment ions of low abundance. CE-ESI-MS analysis of coptisine, berberine and palmatine is shown in Fig. 3. Three peaks were observed in the ion electropherogram of

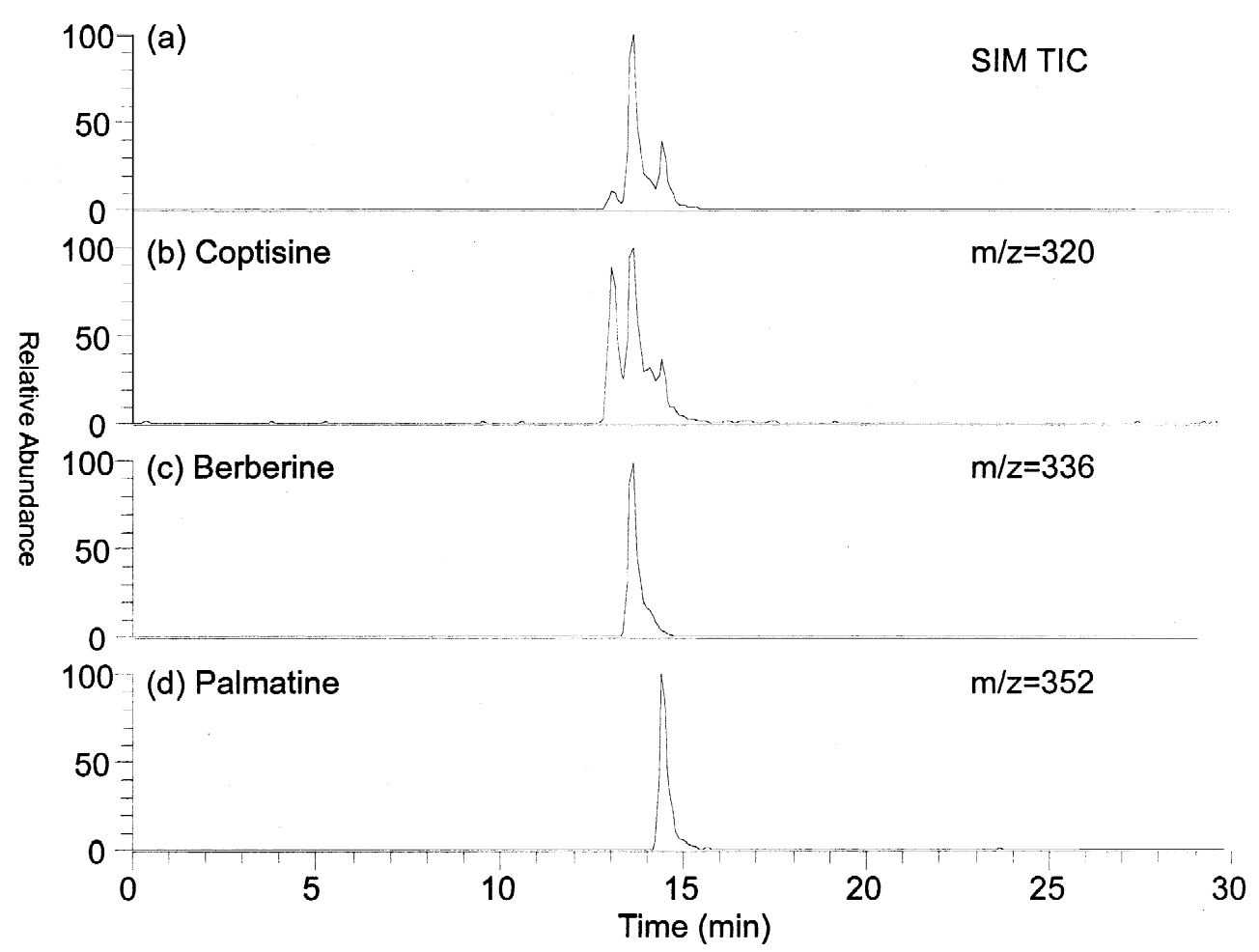

Fig. 4. CE-ESI-MS analysis of Chinese medicine possibly contaminated with coptisine, berberine and palmatine. Conditions as in Fig. 3. 

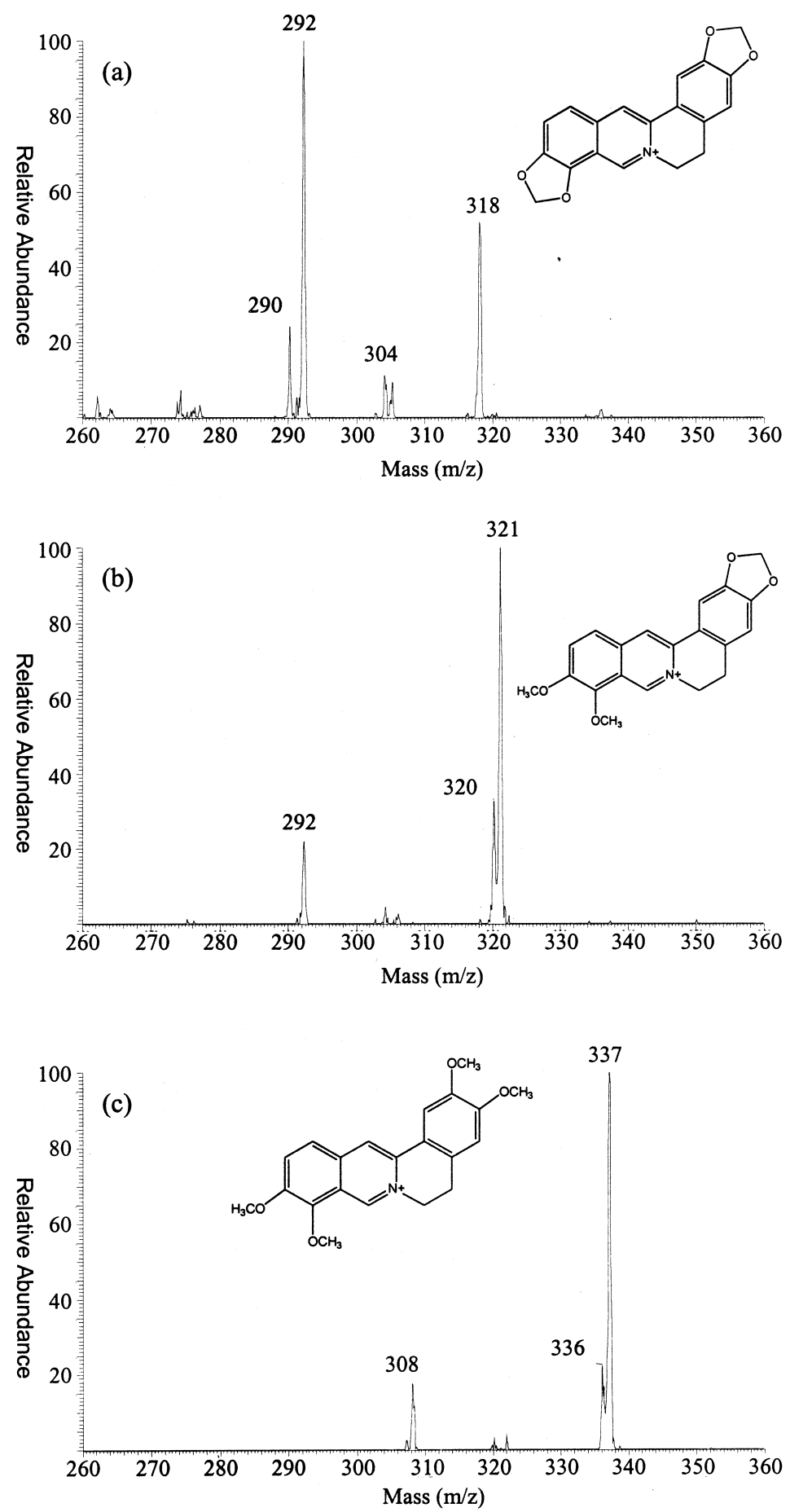

Fig. 5. ESI-MS-MS spectra of (a) coptisine, (b) berberine and (c) palmatine. 
$m / z$ 320. The major peak corresponds to the molecular ion of coptisine and the two small peaks result from the fragmentation of berberine and palmatine. A similar phenomenon was observed in the ion electropherogram of $\mathrm{m} / \mathrm{z} 336$. The major peak is the molecular ion of berberine and the minor peak is the fragment ion of palmatine. Since the retention time may change due to the surface modification, the observation of more than one peak in the ion electropherogram can be a problem in the analysis of these compounds in the crude extract (Fig. 4). In order to overcome the potential interference in the analysis of crude extract, CE was coupled with ESI-MS-MS in the SRM mode.

The ESI-MS-MS spectra of coptisine, berberine and palmatine are shown in Fig. 5. The three most abundant and also characteristic fragment ions were selected for CE-ESI-MS-MS analysis. The analysis of coptisine, berberine and palmatine by CE-ESIMS-MS is shown in Fig. 6. In comparison with the data of CE-ESI-MS, only one peak was observed in the selected ion electropherogram of coptisine, berberine and palmatine. This approach was then applied to the analysis of crude extract and the result is shown in Fig. 7. In comparison with CE-ESI-MS approach (Fig. 4), the trace of coptisine was much cleaner in CE-ESI-MS-MS (Fig. 7).

Based on the data from Fig. 4, berberine appeared to be the major component with palmatine the second and coptisine the least abundant one. These results were inconsistent with the data from $\mathrm{CE}-\mathrm{UV}$ analysis (Fig. 2) in that palmatine was much more abundant than berberine in CE-UV analysis. The relative response of coptisine, berberine and palmatine in UV and ESI were very similar, revealed by the similar relative intensity in Fig. 1 and Fig. 3. This is not unexpected considering the similarity in the structures of coptisine, berberine and palmatine. Because of the very similar UV and ESI sensitivity and the much higher specificity in ESI-MS-MS, the larger peak, which was tentatively identified as palmatine in CE-UV analysis, is very likely con-

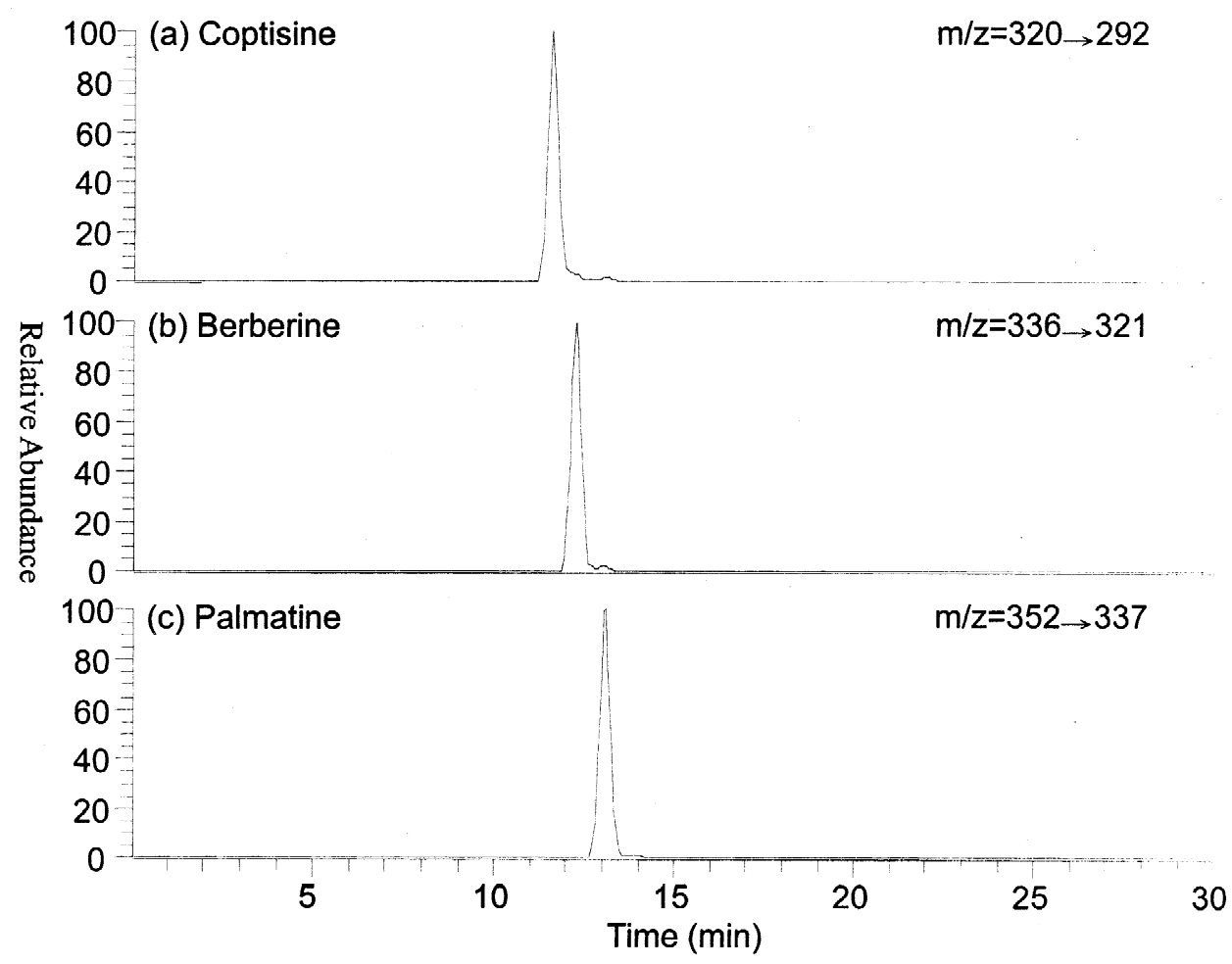

Fig. 6. CE-ESI-MS-MS electropherograms of coptisine, berberine and palmatine. The collision-induced dissociation (CID) fragmentation $m / z=320$ to 292 was selected for coptisine, $m / z=336$ to 321 for berberine and $m / z=352$ to 337 for palmatine. Other conditions as in Fig. 3 . 


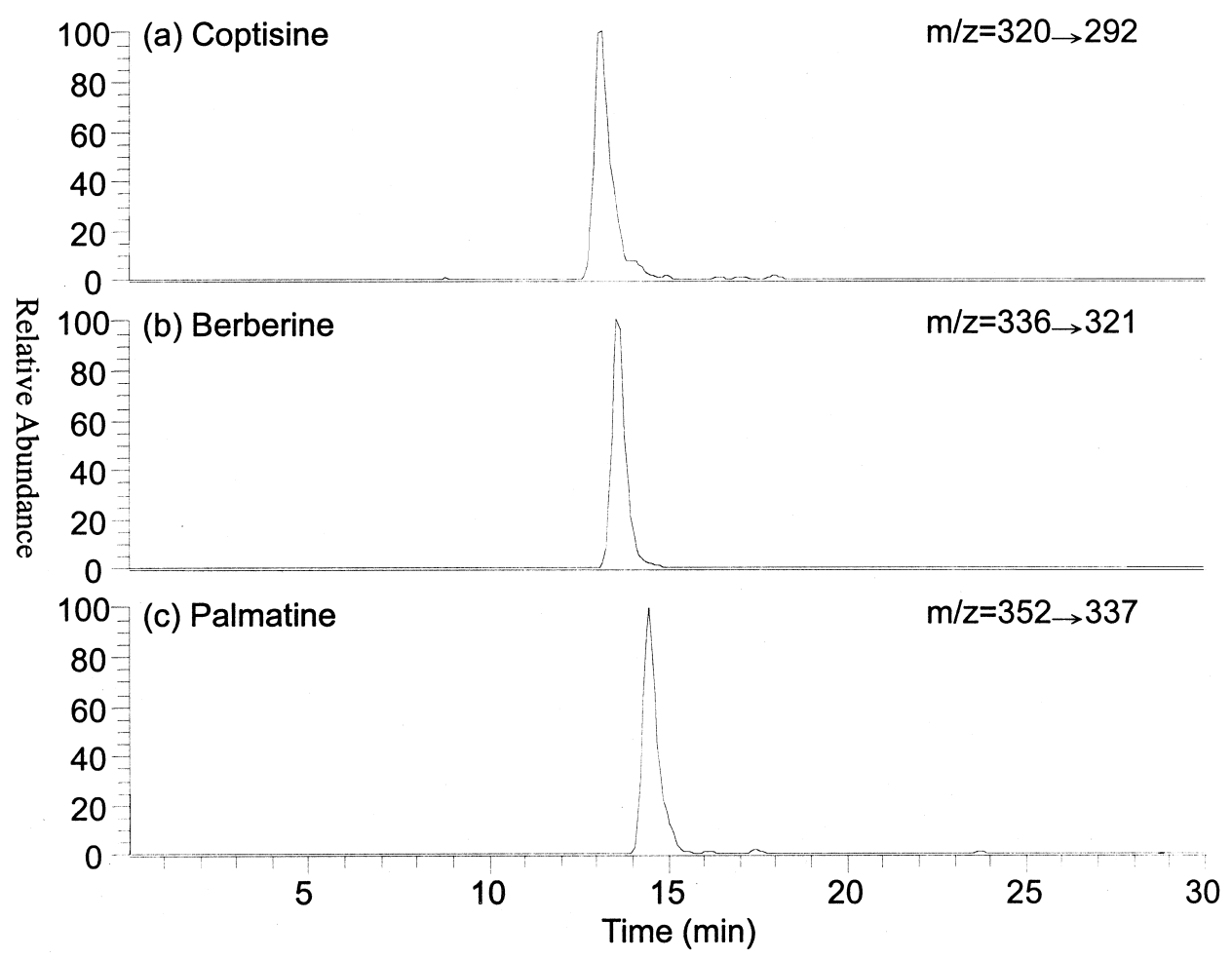

Fig. 7. CE-ESI-MS-MS analysis of the Chinese medicine possibly contaminated with coptisine, berberine and palmatine. Conditions as in Fig. 6.

taining more than one component. Another advantage of using mass spectrometry as the detector is demonstrated in the analysis of the minor component, coptisine. This compound is identified unambiguously by CE-ESI-MS-MS whereas the presence of this compound in the extract is not very clear in CE-UV analysis.

All the herbs listed in wuyoufun-13 had been analyzed and no protoberberine alkaloid was found [36]. The concentrations of coptisine, berberine and palmatine in the sample were $14 \mathrm{ppm}, 50 \mathrm{ppm}$ and $17 \mathrm{ppm}$, respectively. These concentrations are not high enough to have significant medical effect. Considering the price of purchasing the isolated alkaloids and the difficulty of organic synthesis, it is more likely that the sample is contaminated by an herb containing coptisine, berberine and palmatine. Although several Chinese herbs contain protoberberine alkaloids. The herb is believed to be Coptidis rhizoma because only Coptidis rhizoma contains all three alkaloids.

\section{Conclusions}

In this paper, a practical solution of analyzing dubious adulterants coptisine, berberine and palmatine in Chinese medicine "wuyoufun-13" has been demonstrated. Without extensive sample cleaning and preparation, the active ingredients of Coptidis rhisoma in contaminated "wuyoufun-13" were identified by the combination of CE with ESI-MSMS. The use of ESI-MS-MS instead of UV as the detector provides qualitative analysis with higher quality. Furthermore, the problem resulting from the complexity of crude extract such as the complicated electropherogram and poor retention time reproducibility can be overcome by the coupling of $\mathrm{CE}$ with ESI-MS-MS.

\section{References}

[1] M. Tomita, J. Kunitomo, Yakugaku Zasshi 78 (1958) 144. 
[2] O. Ishikawa, T. Hishimoto, T. Nakajima, O. Tanaka, H. Itokawa, Yakugaku Zasshi 98 (1987) 976.

[3] T. Misaki, L. Sagawa, T. Ojima, S. Kakizawa, T. Oshima, H. Yoshizawa, Chem. Pharm. Bull. 30 (1982) 354.

[4] K. Yoneda, E. Yamagata, M. Miyaura, L. Hua, M. Mizuno, Shoyakugaku Zasshi 41 (1987) 205.

[5] K. Yoneda, E. Yamagata, L. Hua, M. Mizuno, Shoyakugaku Zasshi 42 (1988) 116.

[6] L. Zaho, X. Cai, S. Dong, S. Lin, D. An, Zhongguo Yaoke Daxue Xuebao 20 (1989) 82.

[7] G. Luo, Y. Wang, G. Zhou, Y. Yu, J. Liq. Chromatogr. 13 (1990) 3825.

[8] H.L. Lay, H.J. Chan, C.F. Lin, Yaowu Shipin Fenxi 5 (1997) 381.

[9] T.H. Tsai, C.Y. Hong, C.F. Chen, Yaowu Shipin Fenxi 5 (1997) 303.

[10] C.C. Ku, Y.S. Chung, H. Ku, Yaowu Shipin Fenxi 2 (1994) 289.

[11] R. Zheng, G. Li, Zhongcaoyao 24 (1993) 265.

[12] S. Liu, W. Ma, X. Tang, D. Cheng, Zhongcaoyao 25 (1994) 355.

[13] T.C. Wu, S.J. Sheu, Chin. Pharm. J. 45 (1993) 157.

[14] X. Qiu, C. Wu, B. Chen, Yaoxue Xuebao 21 (1986) 458.

[15] S.J. Sheu, C.F. Lu, J. High Resolut. Chromatogr. 19 (1996) 409.

[16] Y.R. Ku, M.J. Tsai, K.C. Wen, Yaowu Shipin Fenxi 3 (1995) 185.

[17] S.J. Sheu, J. Chinese Chem. Soc. 54 (1996) 55.

[18] H.M. Liebich, R. Lehmann, C. Di Stefano, H.U. Haring, J.H. Kim, K.R. Kim, J. Chromatogr. A 795 (1998) 388.
[19] Q. Shen, Z. Fang, Fenxi Shiyanshi 17 (1998) 1.

[20] Y.M. Liu, S.J. Sheu, J. Chromatogr. 639 (1993) 323.

[21] J.L. Wolfender, K. Hostettmann, J. Chromatogr. 647 (1990) 191.

[22] M.A. Aramendia, I. Garcia, F. Lafont, J.M. Marinas, J. Chromatogr. A 707 (1995) 327.

[23] Y. Song, G.R. Her, K.C. Wen, Yaowu Shipin Fenxi 5 (1997) 295.

[24] J. Zeleny, J. Phys. Rev. 10 (1917) 1

[25] M. Dole, R.L. Hines, L.L. Mack, R.C. Mobley, L.D. Ferguson, M.B. Alice, Macromolecules 1 (1968) 96.

[26] M. Yamashita, J.B. Fenn, J. Phys. Chem. 88 (1984) 4451.

[27] M. Yamashita, J.B. Fenn, J. Phys. Chem. 88 (1984) 4671.

[28] J.A. Olivares, N.T. Nguyen, C.R. Yonker, R.D. Smith, Anal. Chem. 59 (1987) 1230.

[29] H.R. Udseth, C.J. Barinaga, R.D. Smith, Anal Chem. 60 (1988) 1948.

[30] E.D. Lee, W. Henion, J.D. Muck, T.R. Covey, J. Chromatogr. 645 (1988) 313.

[31] M.S. Kriger, K.D. Cook, Anal. Chem. 67 (1995) 385.

[32] Y. Takada, M. Sakairi, H. Koizumi, Anal. Chem. 67 (1995) 1474.

[33] T. Sawada, J. Yamahara, K. Goto, M. Yamamura, Yao Hsueh Pao 25 (1971) 74.

[34] J. Haginiwa, M. Harada, Shoyakugaku Zasshi 82 (1962) 726.

[35] C.Y. Tsai, G.R. Her, J. Chromatogr. A 743 (1996) 315.

[36] Thin-Layer Chromatography of Chinese Medicine, Vol. 6, National Laboratories of Food and Drugs, Department of Health, Executive Yuan, Taiwan, 1994. 